\title{
Circuit
}

Musiques contemporaines

\section{Introduction : autour des patenteux}

\section{Jonathan Goldman}

Volume 23, numéro 1, 2013

La musique des objets

URI : https://id.erudit.org/iderudit/1017206ar

DOI : https://doi.org/10.7202/1017206ar

Aller au sommaire du numéro

Éditeur(s)

Les Presses de l’Université de Montréal

ISSN

1183-1693 (imprimé)

1488-9692 (numérique)

Découvrir la revue

Citer ce document

Goldman, J. (2013). Introduction : autour des patenteux. Circuit, 23(1), 5-7.

https://doi.org/10.7202/1017206ar

Ce document est protégé par la loi sur le droit d'auteur. L'utilisation des services d'Érudit (y compris la reproduction) est assujettie à sa politique d'utilisation que vous pouvez consulter en ligne.

https://apropos.erudit.org/fr/usagers/politique-dutilisation/
Cet article est diffusé et préservé par Érudit.

Érudit est un consortium interuniversitaire sans but lucratif composé de l'Université de Montréal, l'Université Laval et l'Université du Québec à Montréal. Il a pour mission la promotion et la valorisation de la recherche. https://www.erudit.org/fr/ 


\section{Introduction : autour des patenteux}

Jonathan Goldman

Le sujet du dossier thématique de ce numéro nous est venu d'un constat: celui d'un rapprochement possible entre les pratiques artistiques d'une masse critique d'artistes sonores québécois des dernières années. Nous disons bien «artiste », et non pas «musicien », car il s'agit d'une pratique entre le domaine habituel de l'art contemporain, sous forme d'installation sonore, et le champ de la musique à proprement parler - si toutefois on peut parler «proprement » d'un phénomène aussi «impur »... Cette pratique engage des objets, souvent arrachés au quotidien et détournés de leur vocation première afin de servir de matériau sonore. Les œuvres ainsi produites constituent des explorations non seulement du potentiel musical de ces objets, mais aussi d'une exposition quasi scientifique - réalisée avec des moyens technologiques tantôt rudimentaires tantôt sophistiqués - de leurs propriétés acoustico-physiques.

On songe dans ce contexte aux cannettes sifflantes de Jean-François Laporte $^{1}$ - dont l'œuvre La plénitude du vide (2005) est au cœur du Cahier d'analyse rédigé ici même par Cléo Palacio-Quintin -, à la symphonie d'imprimantes matricielles de [The User] $(1998)^{2}$, ou encore à l'orchestre de machines à coudre de Martin Messier ${ }^{3}$, pour ne citer que trois exemples caractéristiques que l'on a pu entendre dans la région montréalaise ces dernières années. À l'extérieur de Montréal, des pratiques analogues surgissent avec les Speaker Swinging (haut-parleurs volants; 1982) de l'artiste canadien Gordon Monahan ${ }^{4}$ ou le Conte pour radios et robinets (2004) du Français Arno Fabre5. $\mathrm{Ni}$ compositeurs ou interprètes au sens classique, ni électroacousticiens, ni créateurs de laptop music, ces créateurs se réclament le plus souvent d'une esthétique «DIY» (do it yourself) qui privilégie le lo-fi et la technologie qu'ils sollicitent est même parfois précisément choisie pour son aspect désuet, inactuel. De tels artistes ne forment pourtant pas un groupe homogène et ne se considèrent pas comme appartenant à une tendance esthétique unifiée.

Ce nouveau numéro de Circuit explore donc l'univers inouï de musiciens actifs sur la scène québécoise, canadienne et internationale qui font de la
1. Le lecteur pourra se référer au site web du compositeur:

$<$ www.jflaporte.com> (consulté le 25 mars 2013).

2. Le projet peut être écouté et visionné ici : <www.theuser.org/dotmatrix/fr/ intro.html> (consulté le 25 mars 2013).

3. Certaines photos d'œuvres de Martin Messier ornent le volume 22, $\mathrm{n}^{\circ} 1$ de Circuit. On pourra également consulter le site Internet du compositeurperformeur: <www.mmessier.com> (consulté le 25 mars 2013).

4. Pour une présentation des Speaker Swinging de Monahan, on consultera la page Internet suivante:

$<$ www.gordonmonahan.com/pages/ speaker_swinging.html> (consulté le 25 mars 2013).

5. Une description de l'installation sonore est disponible sur le web: $<$ http://arnofabre.free.fr/ConteRR/ ConteRR.html> (consulté le 25 mars 2013). 
6. De nous avoir suggéré ce rapprochement, nous tenons à remercier Mario Gauthier, grand connaisseur de ce type de production ayant, entre autres, réalisé l'émission "L'espace du son" sur les ondes de Radio-Canada (1996-2001).

7. Un coffret de sept disques consacré à "The Art of David Tudor", paru chez New World Records, permet de prendre la mesure de cet artiste multiforme. Voir: < www.newworldrecords. org/album.cgi?rm=view\&album _ id=91697> (consulté le 13 juin 2013).

8. Voir le portrait de Mario Bertoncini préparé par John Rea dans le vol. 15, no 1 (2004) de Circuit, p. 86-108. musique avec des objets. Souvent présenté sous forme de spectacles dans lesquels les créateurs mettent eux-mêmes en scène les objets dont ils tirent un rendement sonore, parfois à base de mouvements mécaniques amplifiés et/ou traités, il s'agit du travail de créateurs qui tient à la fois de l'instrumental et de l'électroacoustique. Nous les nommons ici "patenteux», sachant qu'il a toujours été dévolu aux critiques musicaux de baptiser les mouvements ou les écoles les plus influents (impressionnistes, atonaux, minimalistes, etc.). Mais hasardons-nous tout de même à donner un nom à cette démarche artistique, le terme de «patenteux» désignant au Québec ceux qui bricolent, détournent, inventent et transforment ainsi leur environnement.

Certes, le mouvement - s'il convient de le caractériser ainsi - n'est pas né ex nihilo. Dans les années 1980 déjà, «Les patenteux du Québec » comptaient parmi ses membres des personnalités-phares de la musique actuelle comme Jean Derome et Pierre Tanguay, qui utilisaient des objets du quotidien comme sources sonores ${ }^{6}$. Il existe également quelques liens évidents à faire avec certaines pratiques de la musique électronique en direct, notamment aux États-Unis dans les années 1960 et 1970, préconisée par des musiciens tels que David Tudor dans, par exemple, les multiples versions de sa Rainforest $(1968-1973)^{7}$, ou Gordon Mumma. Ce qui revient à dire que tout phénomène artistique contemporain - aussi novateur qu'il puisse être - trouve des échos dans des pratiques comparables du passé.

Réfléchir sur ce phénomène actuel implique, bien sûr, de remonter à ses origines possibles. Notre dossier thématique commence ainsi avec un article d'Eric Fillion qui présente l'histoire du groupe Sonde, un ensemble électroacoustique né dans les années 1970 sous l'impulsion des élèves de Mario Bertoncini, professeur invité à la Faculté de musique - aujourd'hui la Schulich School of Music - de l'Université de McGill's. La philosophie du bottega d'arte (l'atelier d'art) de celui-ci favorisait la construction par des compositeurs de leurs propres instruments, ce que Sonde ne manquait pas de faire en proposant l'énigmatique «sahabi », à mi-chemin entre l'instrument de musique et la sculpture sonore: ainsi l'esprit patenteux apparaissait sur la scène, peut-être pour la première fois... Le passage de Bertoncini aura été décisif, mais ce serait falsifier l'histoire que d'attribuer tout un mouvement artistique de fabrication «locale» à l'intervention d'un seul individu.

L'esprit patenteux trouve sans doute en partie son origine dans l'indéniable débrouillardise que l'on trouve chez les gens au Québec. Plus fondamentalement, le «patentisme» est né aussi d'une volonté chez l'artiste-artisan de montrer les moyens de production qu'il emploie: il éprouve un devoir quasi éthique de divulguer au public la source de l'événement sonore et non pas de 
dissimuler ses moyens de production dans une série de douze notes cachée ou dans un patch d'ordinateur; une éthique de la création qui n'est donc pas loin de celle qui a animé autrefois les artistes du Bauhaus.

Quels sont les éléments fondamentaux des pratiques exposées dans ce numéro? Toutes, ou la plupart, abritent un élément «performatif »: l'artiste fait quelque chose sur scène (lancer des «cannettes» ou des haut-parleurs, actionner une machine à coudre, etc.) et le public voit ce qui est fait. Voilà une première différence avec les installations sonores, où l'artiste est souvent absent de l'œuvre. L'art patenteux a donc plus en commun avec le Performance Art tel qu'exécuté par Marina Abramović, dont les New-Yorkais ont pu récemment profiter d'une rétrospective au MoMA9?. De plus, le travail du patenteux a souvent un caractère épistémologique: pour connaître un objet, il faut le faire vibrer, résonner, afin d'entendre et identifier le son qu'il émet.

Dans la suite du numéro, un article du musicologue français Jean-Yves Bosseur répertorie les démarches artistiques de plusieurs créateurs européens de la relève qui, eux aussi, s'intéressent aux potentialités musiciennes des objets arrachés au quotidien. Pour sa part, Lorella Abenavoli dresse un portrait fascinant de Magali Babin, une artiste à la démarche tout à fait unique, très présente sur la scène alternative montréalaise. Nicolas Bernier, artiste sonore, lauréat du prestigieux Prix Ars Electronica 2013, performeur et compositeur, tente, quant à lui, une auto-analyse en relation avec l'héritage légué par la pensée de Pierre Schaeffer et Michel Chion. De plus, et pour la première fois, un document préparé par Mario Gauthier prolonge la thématique de ce numéro en ligne: il s'agit d'un entretien enregistré au printemps 2008 avec Charles de Mestral, membre de Sonde, qui revient sur la pratique avantgardiste du groupe $^{10}$.

Enfin, la rubrique des Actualités s'ouvre sur un dossier réalisé par Serge Provost et Cristina Scuderi sur Re Orso (2012), un opéra avec électronique signé Marco Stroppa. La rubrique est enrichie de la recension d'un livre réalisée par Chantale Laplante, ainsi que de deux comptes rendus autour de films sur l'art par Maxime McKinley (Le chant des ondes, Caroline Martel) et Anne Marie Messier ( $30^{\circ}$ Festival international du Film sur l'Art). Claudine Caron et Cléo Palacio-Quintin ferment le bal avec nos traditionnelles nouveautés en bref.

Bonne lecture!

Montréal, 25 mars 2013
9. Voir la page de l'exposition: $<$ www.moma.org/visit/calendar/ exhibitions/965> (consulté le 25 mars 2013).

10. Disponible sur le site de Circuit: $<$ www.revuecircuit.ca $>$. 